\title{
BMJ Open Protect-me: a parallel-group, triple blinded, placebo-controlled randomised clinical trial protocol assessing antenatal maternal melatonin supplementation for fetal neuroprotection in early-onset fetal growth restriction
}

\author{
Kirsten R Palmer, ${ }^{\circledR 1,2}$ Joanne C Mockler, ${ }^{1,2}$ Miranda L Davies-Tuck, ${ }^{\circledR 3}$ \\ Suzanne L Miller, ${ }^{\oplus, 3}$ Stacy K Goergen,, ${ }^{4,5}$ Michael C Fahey, ${ }^{\oplus 6,7}$ \\ Peter J Anderson, ${ }^{\oplus 8}$ Katie M Groom, ${ }^{\oplus 9}$ Euan M Wallace ${ }^{\oplus 1,3}$
}

To cite: Palmer KR, Mockler JC, Davies-Tuck ML, et al. Protectme: a parallel-group, triple blinded, placebo-controlled randomised clinical trial protocol assessing antenatal maternal melatonin supplementation for fetal neuroprotection in early-onset fetal growth restriction. BMJ Open 2019;9:e028243. doi:10.1136/ bmjopen-2018-028243

- Prepublication history and additional material for this paper are available online. To view these files, please visit the journal online (http://dx.doi. org/10.1136/bmjopen-2018028243).

Received 28 November 2018 Revised 25 March 2019 Accepted 15 May 2019

Check for updates

(c) Author(s) (or their employer(s)) 2019. Re-use permitted under CC BY-NC. No commercial re-use. See rights and permissions. Published by BMJ.

For numbered affiliations see end of article.

Correspondence to Dr Kirsten R Palmer; Kirsten.palmer@monash.edu

\section{ABSTRACT}

Introduction Fetal growth restriction (FGR) is a serious pregnancy complication, associated with increased rates of perinatal death and morbidity among survivors. Most commonly FGR results from placental insufficiency, where the placenta fails to deliver the oxygen and nutrients required for normal fetal growth. This leads to fetal oxidative stress, resulting in organ damage through lipid peroxidation. The early developing brain is particularly susceptible, such that FGR is associated with poorer neurodevelopment, witnessed as cognitive and behavioural dysfunction, and cerebral palsy. Promisingly, melatonin, a lipid soluble antioxidant is neuroprotective in animal models of FGR. We present a protocol outlining a randomised, placebo-controlled trial to explore whether antenatal maternal melatonin supplementation in pregnancies with severe, early-onset FGR can improve neurodevelopment among survivors at 2 years of age.

Methods and analyses We will recruit 336 women with a singleton pregnancy complicated by FGR between 23+0and $31+6$ weeks gestation. Participants will be randomised, stratified by gestational age, to either $30 \mathrm{mg}$ melatonin per day or a visually identical placebo, continued until birth. Measures of maternal and fetal health will be collected until birth. Timing of birth will be determined by the treating clinical team in discussion with the woman. Neonatal and infant neurodevelopmental assessments will be undertaken, consisting of brain MRI at term corrected age, general movements assessment at term and 3 months' corrected age, and Bayley Scales of Infant \& Toddler Development-III and Infant Toddler Social Emotional Assessment at 2.5 years corrected age. Analyses will be on intention to treat. The primary outcome is a difference of 5 points in the cognitive domain of the Bayley-III. Secondary outcomes address maternal and fetal safety.

Ethics and dissemination This trial has Monash Health Human Research and Ethics committee approval (17-0000583A). Findings will be disseminated through peer-reviewed publications, conference presentations and to participants.
Strengths and limitations of this study

- The trial is powered to detect neurodevelopmental performance at 2 years of age from 332 pregnancies, which is a design strength, as assessments performed at an earlier age are less predictive of later neurodevelopmental functioning.

- The secondary outcomes, assessing maternal, fetal and neonatal safety and tolerability, will continue to add to the growing literature regarding the use of melatonin in pregnancy.

- The blinded, randomised, placebo-controlled nature of the trial with stratification to control for the impact of gestational age on neurodevelopmental outcomes strengthens the trial by reducing risks of bias on the results.

- The assessment of multiple developmental domains, including cognition, motor, language and behaviour, as well as structural development will further strengthen our understanding of the impact melatonin may have.

- A limitation of the trial is the current lack of longer-term follow-up, such as to 5-7 years of age, when improved assessment of executive function and other aspects of cognitive performance can be performed; though the trial group intend to maintain contact with the randomised cohort to enable later follow-up.

Trial registration number ACTRN12617001515381; Preresults

\section{INTRODUCTION}

Fetal growth restriction (FGR) is defined as a failure for a fetus to reach its full growth potential. It is a common pregnancy complication, affecting 3\%-9\% of 
pregnancies in high income countries and a reported 30 million babies every year in low income countries. ${ }^{12}$ FGR carries significant mortality risks for the fetus, especially if undetected antenatally. ${ }^{3}{ }^{4}$ Compared with those born at an appropriate weight, survivors of FGR also face increased risks of short- and longterm morbidity, including an increased risk of cardiovascular, renal, endocrine and neurodevelopmental morbidity. ${ }^{5-12}$ With regard to neurodevelopment, FGR is a risk factor for the injury that underlies subsequent cognitive and behavioural impairment, including cerebral palsy. ${ }^{8} 13$

FGR has a variety of aetiologies. The most common cause is placental insufficiency. The relationship of poor placental perfusion with impaired transfer of oxygen and nutrients to the developing fetus and subsequent morbidity is increasingly understood. However, this knowledge provides little comfort to the women with a pregnancy affected by FGR or the clinician at the coal face because current management is limited to preparing for and timing birth to mitigate the risk of stillbirth while balancing the risks of prematurity. ${ }^{14}$ A reality confirmed by the recent outcome of trials exploring sildenafil as a new therapeutic option. ${ }^{15}$ Varying definitions of FGR have hampered research exploring new therapeutic approaches, a problem resolved by the recent development of core outcome sets for FGR that have now produced a consensus definition. ${ }^{16}$ Importantly, this definition also enriches the population studied with FGR due to placental insufficiency, rather than those with a healthy, but small fetus or with FGR due to another aetiological pathway, such as infection or chromosomal anomalies.

For the fetus, placental insufficiency leads to restricted transfer of oxygen and nutrients across the placental bed. The fetus makes a number of compensatory adaptions in this setting, minimising its oxygen consumption and metabolic demands, while also maximising oxygen delivery to its most vital organs, the heart and brain, termed brain sparing. However, the brain is not spared from injury. The brain is particularly vulnerable to the harmful effects of oxidative stress, where reactive oxygen species, mainly through the effects of lipid peroxidation, impair myelination of white matter tracts and cause axonal injury contributing to structural brain changes and the functional impairment seen in postnatal life. ${ }^{13}$

In exploring new therapies that may be applied antenatally to protect the developing fetus from the downstream effects of placental insufficiency, certain qualities are desirable. Such a treatment should be safe, easily administered and well-tolerated by the mother. It should then easily cross the placenta into the fetal circulation, where it needs to be effective, safe and have a wide therapeutic window.

Melatonin is a lipid-soluble hormone capable of crossing the maternal-fetal interface within the placenta. ${ }^{17}$ It is also a potent antioxidant, exerting direct antioxidant effects and upregulating the expression of several antioxidant enzymes, such as superoxide dismutase, catalase and glutathione peroxidase. ${ }^{18} 19$ Importantly, melatonin has been used during pregnancy with no reported adverse effects in the mother. ${ }^{172021}$ In a recently published trial of melatonin given as an adjuvant therapy for pre-eclampsia, there were no apparent significant adverse outcomes to the babies exposed to melatonin antenatally, but there was a higher rate of FGR in those exposed to melatonin compared with historic controls. ${ }^{20}$ In an ovine model of FGR, melatonin protects the brain from the harmful effects of oxidative stress, minimises structural brain injuries in the lambs and improves their neurodevelopmental performance. ${ }^{21}$ Furthermore, in a phase one clinical trial of melatonin in human pregnancies complicated by FGR, placental oxidative stress was significantly reduced in those exposed to melatonin. ${ }^{21}$

\section{Rationale}

Placental insufficiency leading to FGR is relatively common and a significant risk factor for adverse fetal outcomes during pregnancy that also lays the foundation for a multitude of lifelong adverse health outcomes for the child and their family. Damage to the developing fetal brain occurs antenatally due to chronic hypoxia, inflammation and oxidative stress, and accordingly, any neuroprotective intervention must be implemented during pregnancy to prevent brain injury. The impact that placental insufficiency and FGR can have on the developing brain is profound, with children and adults having higher rates of behavioural, cognitive, motor and language dysfunction, and an elevated risk of cerebral palsy. ${ }^{5132223}$ The maternal use of melatonin, an anti-oxidant that can easily cross the placenta, may provide protection to the fetal brain from the damaging effects of reactive oxygen species leading to less neurological injury and improved neurodevelopmental performance in childhood.

\section{Aims}

The trial is designed to test the hypothesis that antenatal maternal melatonin supplementation improves neurodevelopmental outcomes in children affected by FGR. The primary aim is to determine whether neurodevelopment at 2.5 years corrected age ( \pm 6 months) is enhanced among survivors of FGR whose mothers receive melatonin antenatally compared with those whose mothers receive placebo.

Secondary aims will seek to provide further fetal and maternal safety data on the use of melatonin in pregnancy. Specifically, we aim to:

1. To determine the effects of melatonin supplementation on fetal growth and ultrasound measures of the utero-placental-fetal circulation.

2. To report the occurrence of any adverse and serious adverse events (AEs) associated with melatonin use.

\section{METHODS AND ANALYSIS \\ Study design}

A multicentre, triple-blind, randomised, parallel group, placebo-controlled trial developed in accordance with 
Table 1 Standard Protocol Items: Recommendations for Interventional Trials trial data

\section{Data category}

Primary registry and trial ID

Date of registration in primary registry

Secondary identifying numbers

Source of funding or material support

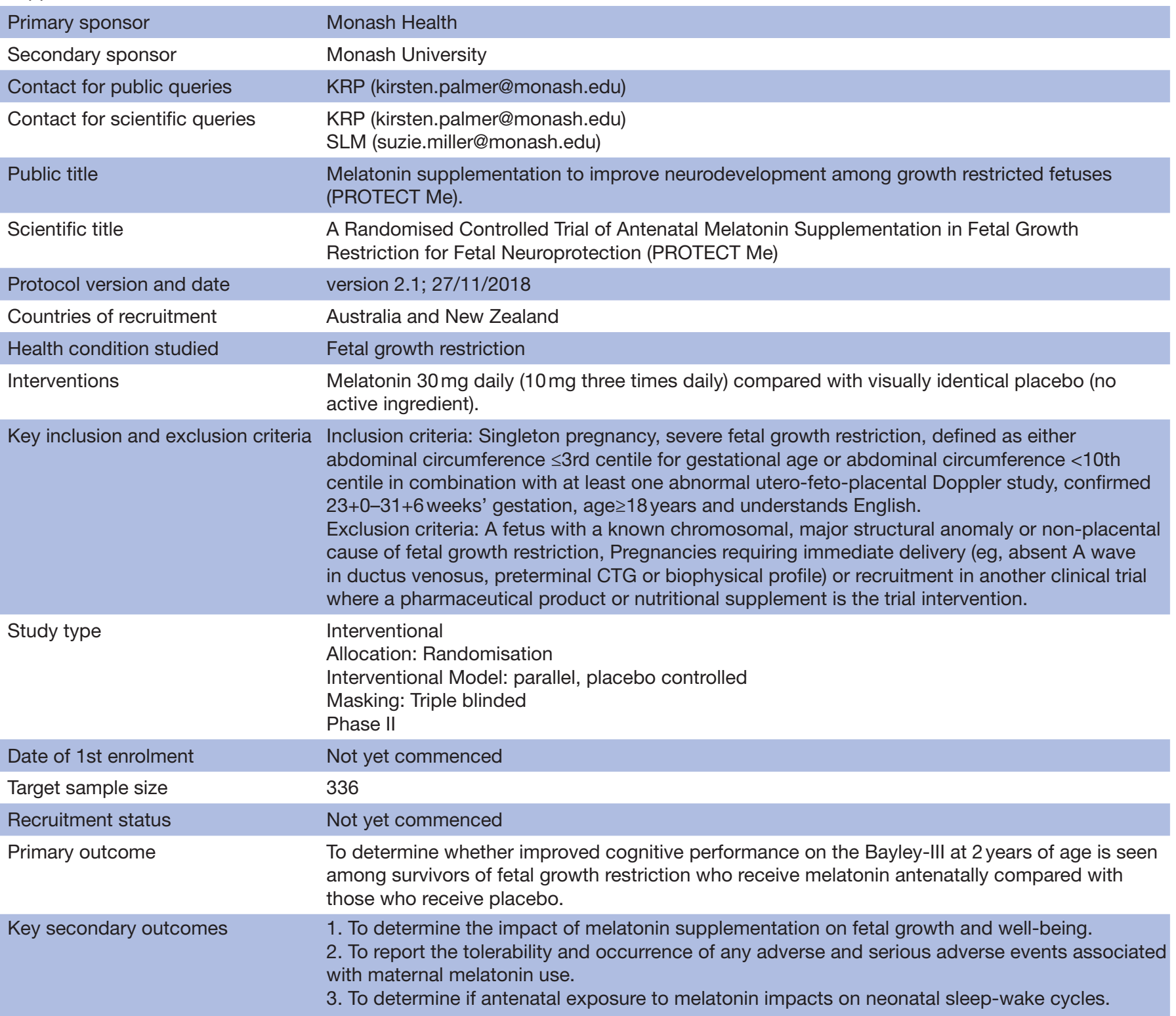

Standard Protocol Items: Recommendations for Interventional Trials guidelines (see table 1$).^{24}$

\section{Study setting}

Trial recruitment will be undertaken through maternal fetal medicine and tertiary obstetric units located in major maternity referral hospitals across Australia and New Zealand. Participating sites are required to have access to MRI equipment needed for the trial protocol. These centres are chosen as they are the main sites of care for these complicated pregnancies.

\section{Subjects}

Pregnant women with a singleton pregnancy complicated by severe FGR identified at 23+0-31+6 weeks' gestation.

\section{Inclusion criteria}

1. Singleton pregnancy.

2. Severe FGR, defined as:

1. Abdominal circumference $\leq 3 r d$ centile for gestational age by adapted Westerway et al charts ${ }^{25}$ or 
2. Abdominal circumference $<10$ th centile ${ }^{25}$ in combination with at least one abnormal fetoplacental Doppler study, being:

1. Uterine artery (raised pulsatility index $\geq 95$ th centile). ${ }^{26}$

2. Umbilical artery (pulsatility index $\geq 95$ th centile $^{27}$ or absent/reversed end-diastolic flow).

3. $23+0-31+6$ weeks' gestation.

4. Age $\geq 18$ years.

5. Understands English.

\section{Exclusion criteria}

1. A fetus with a known chromosomal, major structural anomaly or non-placental cause of FGR.

2. Pregnancies requiring immediate delivery (eg, absent A wave in ductus venosus, preterminal Cardiotocograph (CTG) or biophysical profile).

3. Co-recruitment in another clinical trial where a pharmaceutical product or nutritional supplement impacting on oxidative stress is the trial intervention.

\section{Recruitment}

Potential trial participants will be identified from referrals to maternal fetal medicine units, tertiary obstetric units or perinatal ultrasound services at participating sites. Eligible women will be approached by a member of the research team who is not involved in their care and provided with the Participant Information and Consent Form for the trial (see online supplementary appendix 1). The research team member will give a verbal explanation of the trial, including a description of the trial processes, the voluntary nature of the trial and that a decision to participate, or not, will not affect standard clinical care.

Women who provide written informed consent to participate will then be randomised to receive either melatonin (EuroVital pharmaceuticals, Scottsdale, Arizona, USA) or a matched placebo (PCI pharma services, Port Melbourne, Victoria, Australia).

\section{Randomisation}

Randomisation will be via an on-line computerised randomisation service. Participants will be stratified depending on their gestational age at recruitment, producing two groups, those between $23+0-27+6$ weeks' gestation and those 28+0-31+6 weeks' gestation. Within these gestational groups, participants will be further stratified for the presence or absence of abnormal umbilical Dopplers then randomised in a 1:1 ratio to melatonin or placebo. Stratification for gestational age and Dopplers is to better control for the effect that both preterm birth and abnormal Dopplers can have on subsequent neurodevelopmental outcomes. ${ }^{23}$ At the time of recruitment, each participant will be assigned a unique trial code. Subsequently, all data and tissue samples collected from the participant will be stored only with this associated code. Thus, all data and tissue samples are de-identified at the point of collection, but with the capability to re-identify them in the future for data verification, research audit and safety (eg, needlestick injury). A repository of all de-identified human tissue samples collected during the trial will be maintained at the Monash Health Translation Precinct and access to these samples overseen by the Monash Health Human Research Ethics Committee.

\section{Trial intervention}

After randomisation, participants will commence the trial intervention, of either melatonin $10 \mathrm{mg}$ slow release tablets (EuroVital pharmaceuticals, Scottsdale, Arizona, USA), administered three times a day (a total daily dose of $30 \mathrm{mg}$ per day) or visually identical placebo tablets containing no active ingredient, administered three times a day. Trial participants will continue the trial intervention until delivery.

Participants, their healthcare providers and the research team will be blinded to the trial intervention the participant has received until all participants have completed all the trial stages.

The trial intervention will be stored at each recruiting site according to local hospital pharmacy procedure. It will be dispensed in bottles containing 2 weeks supply (42 tablets). Compliance will be assessed by a review of a participant medication diary and tablet count on return of each trial intervention bottle.

\section{Outcomes}

\section{Primary outcome}

Performance in the cognitive domain of the Bayley-III at 2.5 years corrected age ( \pm 6 months).

\section{Secondary outcomes}

Address the safety of melatonin use within this population for both the mother, as well as the fetus and neonate. This will be achieved through:

- Assessment of fetal growth through antenatal fetal ultrasound surveillance of growth, as well as collection of birth weight data.

- Assessment of fetal well-being through the measure of uteroplacental and fetal Doppler indices, being the pulsatility index of the uterine arteries, umbilical artery, middle cerebral artery and ductus venosus, as well as the amniotic fluid index.

- Neonatal observation through the use of a sleepwake-fuss chart completed hourly during the first week of life.

- Assessment of drug safety and tolerability for the mother will be measured through the use of a participant medication diary, as well as haematological and biochemical tests.

- Assessment of melatonin concentration in maternal and fetal circulations.

\section{Sample and data collection}

Data for each participant will be collected at recruitment, including age, self-reported ethnicity, height and weight, body mass index, past obstetric history (eg, parity and 
previous pregnancy complications, such as FGR, pre-eclampsia, preterm birth or gestational diabetes), past medical history (eg, pre-existing renal, cardiovascular or autoimmune disease), current medication use, method of estimating due date and gestational age in current pregnancy, presence of other obstetric complications in current pregnancy, aneuploidy screening and, if done, results from invasive diagnostic tests and fetal infection screens.

Following randomisation participants will have blood pressure and urinalysis (dipstick for proteinuria) measured fortnightly to monitor for evolving pre-eclampsia, as well as information about any hospital admissions or concomitant development of pre-eclampsia. To ensure maternal safety, full blood count, renal (urea, electrolytes, creatinine, urate) and liver (Alanine transaminase (ALT), bilirubin, albumin) function will be tested before the commencement of treatment, 48 hours \pm 24 hours and 14 days ( \pm 2 days) after initiation of trial medication, on the day of delivery and then 1 week ( \pm 3 days) after birth. Results from these tests will be available to the treating clinical team. Umbilical cord blood and maternal serum will be collected at the time of delivery for assessing melatonin concentrations in the fetal and maternal circulations respectively. All blood samples collected at delivery for assessing melatonin concentrations will be centrifuged at $2500 \mathrm{~g}$ for 20 min to obtain serum for aliquoting prior to storage at $-80^{\circ} \mathrm{C}$ until the time of analysis. These results will not be available to the treating team.

Ultrasound assessment of fetal growth and well-being, in keeping with standard operating procedures that will be provided to all participating sites to limit variability, will be performed fortnightly until birth for trial purposes. Results will be available to the treating team to guide clinical decision-making. Scans may be performed more frequently at the discretion of the treating clinical team.

Following birth, data for both maternal and neonatal birth outcomes will be collected to the time of hospital discharge from medical records. A series of neurodevelopmental assessments will also be performed (schedule outlined in figure 1). These include an infant MRI at term corrected age, performed with a 3T MRI scanner, such as Philips Ingenia (Amsterdam, Netherlands) or similar. Scanning will be performed using a non-sedated 'wrap and feed' technique. High-resolution axial imaging using T1, T2 and DWI sequences will be obtained. Diffusion tensor imaging will be used to study white matter tract microstructure. This technique allows calculation of fractional anisotropy, axial diffusion and radial diffusion of cerebral white matter tracts. All MRI studies will be reported by a single neuroradiologist blinded to treatment group. The MRI results will be available to the treating team to guide clinical decision-making.

A general movements assessment (GMA) will be performed at term and at 3 months' corrected age \pm 2 weeks. The GMA is a non-invasive, quantifiable assessment of spontaneous infant movement. Episodes of spontaneous movements are assessed via video recordings using established methodology, ${ }^{28}$ with movements classified as normal, abnormal or absent depending on the gestational age of the infant and number of weeks post birth. The GMA is a clinically useful indicator of an individual's risk for developing cerebral palsy with high positive and negative predictive values. ${ }^{29}$ All GMAs will be performed by assessors blinded to treatment group and results will be available to the treating team. Additional interventional care will be offered if indicated. The research team will not be involved in this care nor in the decisions regarding the need for interventional therapy.

At 2.5 years corrected age $( \pm 6$ months $)$ the Bayley-III and ITSEA will be performed. We will administer the cognitive, language and motor domains of the Bayley-III. The Bayley-III is a direct assessment of neurodevelopment and will be conducted by an experienced developmental psychologist blinded to (1) treatment group and (2) GMA scores. The ITSEA assesses a wide array of

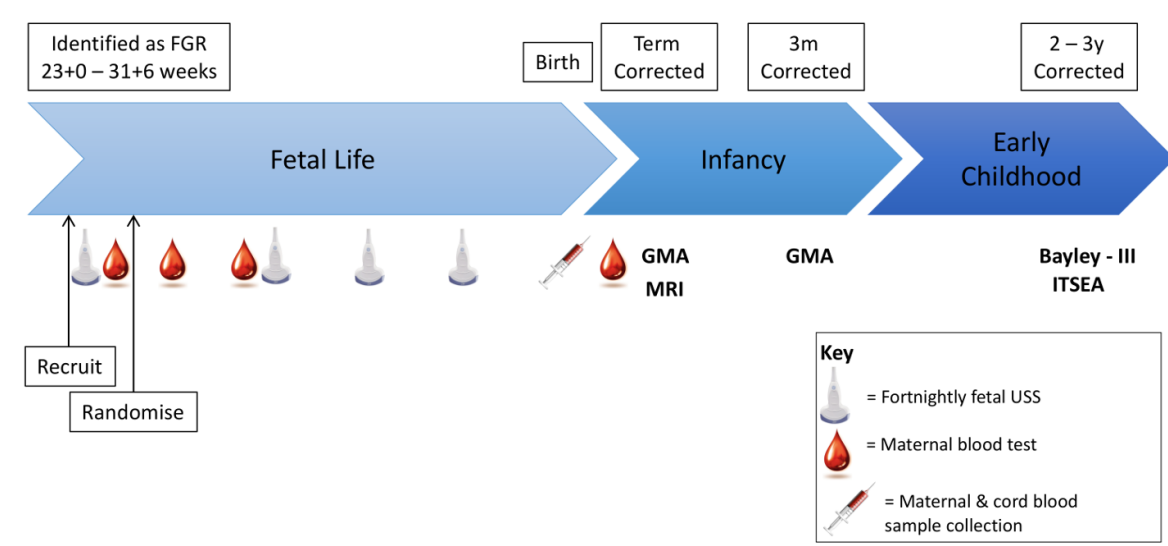

Figure 1 Individual patient trial schedule. During fetal life, maternal blood will be collected at recruitment, 48 hours and 14 days postcommencement of the trial intervention, at birth and 1 week postpartum. Fortnightly fetal ultrasound assessments of growth and well-being will occur. Postnatal assessments include the GMA, MRI, Bayley III scales of infant and toddler development (Bayley-III) and ITSEA as outlined. 3m, 3 months; 2-3y, 2-3 years. FGR, fetal growth restriction; GMA, general movements assessment; ITSEA, Infant Toddler Social Emotional Assessment; USS, ultrasound scan. 
social-emotional and behavioural problems and competencies. It is validated for use between 12-36 months of age and will be applied to our trial cohort at 24-36 months corrected age. It is a parent-reported assessment undertaken in the form of a questionnaire. Results will be evaluated by an experienced developmental psychologist blinded to treatment group, GMA and Bayley-III data. Any concerning results from either assessment will be discussed with the family and where appropriate referral onto appropriate specialists/services made.

All data collected through-out the trial will be stored in a secure, password protected REDCap database. Access to the database will only be available to the principal investigator and research team involved in data entry and analysis.

\section{Sample size}

The sample size has been calculated to detect if melatonin supplementation affords a clinically relevant difference in neurodevelopmental outcomes among survivors overall, as well as being powered to detect this difference within subgroups of fetuses affected by very early- or early-onset FGR. On average, the growth restricted infant has a cognitive score five points lower (with a SD of 9) than the healthy preterm infant and eight points lower than the healthy term infant. ${ }^{30}$ An increase of $4-5$ points in the Bayley-III Cognitive scale has been deemed sufficiently clinically meaningful to drive changes in health policy previously. ${ }^{31}$ Therefore, we have chosen 4-5 points difference on the Bayley-III to power the effect size. There are no data to date to suggest melatonin will have a different effect size based on very early $(<28$ weeks) versus early-onset FGR $(28-31+6$ weeks), but we expect neurocognitive outcomes to differ by gestation at birth, as those identified with FGR before 28 weeks' gestation are known to have greater morbidity and mortality. ${ }^{32}{ }^{33}$ With the very early-onset group likely to deliver preterm, we will recruit babies equally within each group to account for this potential interaction. Therefore with $90 \%$ power, two-tailed, alpha level $<0.05$ we need 69 mother-baby pairs per group.
Assuming a 5\% loss to follow-up rate, we aim to recruit an extra 14 participants overall. Furthermore, a perinatal loss rate of $\sim 15 \%$ is commonly observed among pregnancies complicated by FGR diagnosed $<32$ weeks' gestation. ${ }^{34}{ }^{35}$ Allowing for this, an extra 44 women will be recruited overall. Therefore, each group will have an additional 15 women recruited giving a total of 84 mother-baby pairs per group and a total trial population of 336 mother-baby pairs (see figure 2 ).

\section{Proposed analysis}

Participant data will be analysed by intention to treat. All continuous data will be assessed for normality. Continuous data will be described using mean (SD) if normally distributed and median ( $\mathrm{IQR}$ ) when the distribution is skewed. Dichotomous outcomes will be presented as risk ratios with $95 \%$ confidence intervals using $\chi^{2}$ analysis for significance testing. In the event of a value less than five, Fisher's Exact test will be used. Differences in maternal and pregnancy characteristics between the two arms and within each gestational age strata will be compared using standard techniques to assess the effectiveness of randomisation. Differences in the primary outcome, Bayley-III score at 2.5 years corrected age ( \pm 6 months), and secondary outcomes (fetal growth, fetal and uteroplacental Dopplers, neonatal sleep-wake cycles, maternal side effects and drug compliance, and melatonin concentrations in maternal and fetal blood) will be compared between the two arms. Multivariable regression adjusting for potential imbalances of confounders between the two groups will be performed if relevant. A planned sub-group analysis comparing primary and secondary outcomes stratified by gestational group will also be performed. A p value $<0.05$ (two-tailed) will be considered statistically significant.

\section{Adverse events}

While no clinical studies have demonstrated any serious adverse reactions to melatonin supplements in pregnancy, ${ }^{172021}$ we cannot rule out the possibility of

Figure 2 Flow chart of treatment groups. FGR, fetal growth restriction. 
unexpected adverse reactions. Accordingly, the site investigators will monitor trial participants and their babies progress closely, as well as being contactable to participants by phone at all times. AE assessment and reporting will be undertaken in line with the requirements of the Sponsor, Monash Health and the National Health Medical Research Council (NHMRC). All observed or volunteered AEs/serious AEs (SAEs) regardless of causal relationship will be recorded and reported in detail in participant medical records, to the Monash Health Human Ethics Committee and the Sponsor, Monash Health within 24-72 hours.

\section{Data and safety monitoring committee reporting}

Melatonin is not currently indicated as an antenatal fetal neuroprotective agent during pregnancies with FGR, therefore for reasons of participant and fetal safety, a data and safety monitoring committee (DSMC) has been established. The DSMC will consist of two Australian Health Professional Regulatory Agency registered medical doctors, being a neonatologist and an obstetrician, as well as a biostatistician. Two members of the DSMC have clinical trials experience and have participated as a member of a DSMC before.

For reasons of safety, the DSMC will review de-identified (but potentially re-identifiable) data on the following:

- Interim data for evidence of trial-related AEs.

- Data quality, completeness and timeliness.

- Adherence to trial protocol.

- Adequacy of compliance with goals for recruitment and retention.

- Factors that might affect trial outcome:

Compromise of confidentiality of data, such as protocol violations, unblinding and so on.

Scientific or therapeutic advances that may impact on participant safety or the ethics of the study.

The DSMC are advised to advocate complete cessation, or re-evaluation, of the trial conduct, if it is evident that either arm of the trial is associated with a statistically significant increase in or a $50 \%$ increased rate above the baseline ratio of trial-related AEs.

The DSMC will review trial data after $30 \%(\mathrm{n}=100)$, and $60 \%(n=200)$ of trial participants have been recruited.

\section{Trial discontinuation or modification}

Participants may elect to withdraw their participation from either a specified component of (eg, cessation of trial intervention, but participate in neurodevelopmental follow-up), or the full trial. Otherwise their continued participation may be withdrawn at the request of the principal investigator responsible for trial-related medical decisions. For example, following a SAE or AE that is assessed by the PI, as being directly related to the administration of the trial intervention or as a consequence of trial participation. The trial may be discontinued at the request of the human research ethics committee/research directorate or at the request of the DSMC.
There will be no allowance for the modification of the trial intervention. Where necessary any modifications to the trial protocol would require approval from the Monash Health Human Research Ethics Committee.

\section{Patient and public involvement}

The trial design, rationale and planned intervention has been discussed and planned with consumers to ensure acceptability. Women approached for recruitment will be asked to complete a short questionnaire to provide feedback on their concerns relating to FGR and the nature of antenatal interventions they would accept. Those participating in the trial will also be asked for feedback on the trial intervention, the trial process and any difficulties encountered. Information regarding their attitudes towards the implementation of trial findings if positive will also be sought. Trial participants will receive financial assistance towards travel-related expenses associated with the performance of the Bayley-III and ITSEA at 2.5 years \pm 6 months corrected age.

\section{ETHICS AND DISSEMINATION}

Trial findings will be disseminated through presentations at national and international conferences, publication in peer reviewed journals, as well as digital and print media. Publication authorship will be granted by the principal investigators to those who have contributed to the work in the capacities defined by the NHMRC ${ }^{36}$ and specific journal guidelines regarding the attribution of authorship. Results will also be shared with all the trial participants. Consent from trial participants will also be sought to enable sharing of de-identified data to assist with individual patient data meta-analyses with similarly planned international trials. Participants will also be asked for their consent to be contacted for future follow-up studies.

\section{DISCUSSION}

Severe early-onset FGR is a known contributor to structural brain injury and neurodevelopmental morbidity due to the effects of oxidative stress suffered during the antenatal period. No therapies currently exist that can modify this course of events. Melatonin, a potent antioxidant, able to freely traverse the placental bed from the maternal to the fetal circulation may show benefit in ameliorating the effects of placental insufficiency on brain development, as it has done in animal models of FGR. ${ }^{21}{ }^{37}$ If so, melatonin would be the first therapy capable of reducing the morbidity of severe FGR and would likely be rapidly implemented into standard practice for the care of these highly challenging pregnancies. As neurodevelopment continues through-out childhood and adolescence, it is anticipated however that longer term follow-up beyond that outlined in this trial is a necessity to appreciate the impact that melatonin may be able to provide for long term neurodevelopmental outcomes. 
Author affiliations

${ }^{1}$ Department of Obstetrics and Gynaecology, Monash University, Clayton, Victoria, Australia

${ }^{2}$ Department of Obstetrics and Gynaecology, Monash Health, Clayton, Victoria, Australia

${ }^{3}$ The Ritchie Centre, Hudson Institute of Medical Research, Clayton, Victoria, Australia

${ }^{4}$ Department of Imaging, Monash University, Clayton, Victoria, Australia

${ }^{5}$ Department of Radiology, Monash Health, Clayton, Victoria, Australia

${ }^{6}$ Department of Paediatric Neurology, Monash Health, Clayton, Victoria, Australia

${ }^{7}$ Department of Paediatrics, Monash University, Clayton, Victoria, Australia

${ }^{8}$ Monash Institute of Cognitive and Clinical Neuroscience, Monash University,

Clayton, Victoria, Australia

${ }^{9}$ Department of Obstetrics and Gynaecology, Liggins Institute, University of Auckland, Auckland, New Zealand

Contributors The original idea for the trial was generated by EW and SLM. KRP developed the trial protocol with assistance from JM, KMG, PJA, MCF, SKG MD-T and EW. KRP drafted the protocol for publication and all authors provided contributions to editing and approval of the final manuscript.

Funding The trial is supported by funding from the Cerebral Palsy Alliance (Medical Research Future Fund) and the Equity Trustee's Lynn Quayle Charitable Trust. KRP is funded by a Monash University School of Clinical Sciences Practitioner Fellowship and by the David Healy Clinical Research Fellowship.

Competing interests None declared.

Patient consent for publication Not required.

Ethics approval This trial has received ethics approval from the Monash Health Human Research Ethics Committee (17-0000-583A).

Provenance and peer review Not commissioned; externally peer reviewed.

Data sharing statement Consent from trial participants will also be sought to enable sharing of de-identified data to assist with individual patient data metaanalyses with similarly planned international trials.

Open access This is an open access article distributed in accordance with the Creative Commons Attribution Non Commercial (CC BY-NC 4.0) license, which permits others to distribute, remix, adapt, build upon this work non-commercially, and license their derivative works on different terms, provided the original work is properly cited, appropriate credit is given, any changes made indicated, and the use is non-commercial. See: http://creativecommons.org/licenses/by-nc/4.0/.

\section{REFERENCES}

1. de Onis M, Blössner M, Villar J. Levels and patterns of intrauterine growth retardation in developing countries. Eur J Clin Nutr 1998;52(Suppl 1):S5-15.

2. Figueras F, Gratacós E. Update on the diagnosis and classification of fetal growth restriction and proposal of a stage-based management protocol. Fetal Diagn Ther 2014;36:86-98.

3. Gardosi J, Madurasinghe V, Williams M, et al. Maternal and fetal risk factors for stillbirth: population based study. BMJ 2013;346:f108.

4. Figueras F, Gardosi J. Intrauterine growth restriction: new concepts in antenatal surveillance, diagnosis, and management. Am J Obstet Gynecol 2011;204:288-300.

5. Levine TA, Grunau RE, McAuliffe FM, et al. Early childhood neurodevelopment after intrauterine growth restriction: a systematic review. Pediatrics 2015;135:126-41.

6. Crispi F, Miranda J, Gratacós E. Long-term cardiovascular consequences of fetal growth restriction: biology, clinical implications, and opportunities for prevention of adult disease. Am J Obstet Gynecol 2018;218:S869-S879.

7. von Beckerath AK, Kollmann M, Rotky-Fast C, et al. Perinatal complications and long-term neurodevelopmental outcome of infants with intrauterine growth restriction. Am J Obstet Gynecol 2013;208:130.e1-130.e6.

8. Baschat AA. Neurodevelopment after fetal growth restriction. Fetal Diagn Ther 2014;36:136-42.

9. Ojeda NB, Grigore D, Alexander BT. Intrauterine growth restriction: fetal programming of hypertension and kidney disease. Adv Chronic Kidney Dis 2008;15:101-6.

10. Wang YP, Chen X, Zhang ZK, et al. Increased renal apoptosis and reduced renin-angiotensin system in fetal growth restriction. $J$ Renin Angiotensin Aldosterone Syst 2016;17:17.
11. Salam RA, Das JK, Bhutta ZA. Impact of intrauterine growth restriction on long-term health. Curr Opin Clin Nutr Metab Care 2014;17:249-54.

12. Yzydorczyk C, Armengaud JB, Peyter AC, et al. Endothelial dysfunction in individuals born after fetal growth restriction: cardiovascular and renal consequences and preventive approaches. J Dev Orig Health Dis 2017;8:448-64.

13. Miller SL, Huppi PS, Mallard C. The consequences of fetal growth restriction on brain structure and neurodevelopmental outcome. J Physiol 2016;594:807-23.

14. Groom KM, David AL, Chb MB. The role of aspirin, heparin, and other interventions in the prevention and treatment of fetal growth restriction. Am J Obstet Gynecol 2018;218:S829-S840.

15. Groom KM, Ganzevoort W, Alfirevic Z, et al. Clinicians should stop prescribing sildenafil for fetal growth restriction (FGR): comment from the STRIDER Consortium. Ultrasound Obstet Gynecol 2018;52:295-6.

16. Gordijn SJ, Beune IM, Thilaganathan B, et al. Consensus definition of fetal growth restriction: a Delphi procedure. Ultrasound Obstet Gynecol 2016;48:333-9.

17. Okatani $\mathrm{Y}$, Okamoto K, Hayashi K, et al. Maternal-fetal transfer of melatonin in pregnant women near term. J Pineal Res 1998;25:129-34.

18. Reiter RJ, Tan DX, Osuna C, et al. Actions of melatonin in the reduction of oxidative stress. A review. J Biomed Sci 2000;7:444-58.

19. Galano A, Reiter RJ. Melatonin and its metabolites vs oxidative stress: From individual actions to collective protection. J Pineal Res 2018;65:e12514.

20. Hobson SR, Gurusinghe S, Lim R, et al. Melatonin improves endothelial function in vitro and prolongs pregnancy in women with early-onset preeclampsia. J Pineal Res 2018;65:e12508.

21. Miller SL, Yawno T, Alers NO, et al. Antenatal antioxidant treatment with melatonin to decrease newborn neurodevelopmental deficits and brain injury caused by fetal growth restriction. J Pineal Res 2014;56:283-94.

22. Baschat AA, Viscardi RM, Hussey-Gardner B, et al. Infant neurodevelopment following fetal growth restriction: relationship with antepartum surveillance parameters. Ultrasound Obstet Gynecol 2009;33:44-50.

23. Murray E, Fernandes M, Fazel M, et al. Differential effect of intrauterine growth restriction on childhood neurodevelopment: a systematic review. BJOG 2015;122:1062-72.

24. Chan AW, Tetzlaff JM, Gøtzsche PC, et al. SPIRIT 2013 explanation and elaboration: guidance for protocols of clinical trials. BMJ 2013;346:e7586-42

25. Westerway SC, Davison A, Cowell S. Ultrasonic fetal measurements: new Australian standards for the new millennium. Aust N Z J Obstet Gynaecol 2000;40:297-302.

26. Gómez O, Figueras F, Fernández S, et al. Reference ranges for uterine artery mean pulsatility index at 11-41 weeks of gestation. Ultrasound Obstet Gynecol 2008;32:128-32.

27. Acharya G, Wilsgaard T, Berntsen GK, et al. Reference ranges for serial measurements of umbilical artery Doppler indices in the second half of pregnancy. Am J Obstet Gynecol 2005;192:937-44.

28. Einspieler C, Prechtl HFR, Ferrari F, et al. The qualitative assessment of general movements in preterm, term and young infants - Review of the methodology. Vol. 50, Early Human Development: Elsevier, 1997:47-60.

29. Adde L, Rygg M, Lossius K, et al. General movement assessment: predicting cerebral palsy in clinical practise. Early Hum Dev 2007:83:13-18.

30. Padilla N, Falcón C, Sanz-Cortés M, et al. Differential effects of intrauterine growth restriction on brain structure and development in preterm infants: a magnetic resonance imaging study. Brain Res 2011;1382:98-108.

31. Makrides M, Gibson RA, McPhee AJ, et al. Neurodevelopmental outcomes of preterm infants fed high-dose docosahexaenoic acid: a randomized controlled trial. JAMA 2009;301:175-82.

32. Lees C, Marlow N, Arabin B, et al. Perinatal morbidity and mortality in early-onset fetal growth restriction: cohort outcomes of the trial of randomized umbilical and fetal flow in Europe (TRUFFLE). Ultrasound Obstet Gynecol 2013;42:400-8.

33. Monier I, Ancel PY, Ego A, et al. Gestational age at diagnosis of early-onset fetal growth restriction and impact on management and survival: a population-based cohort study. BJOG 2017;124:1899-906.

34. Cruz-Lemini M, Crispi F, Van Mieghem T, et al. Risk of perinatal death in early-onset intrauterine growth restriction according to gestational age and cardiovascular Doppler indices: a multicenter study. Fetal Diagn Ther 2012;32:116-22. 
35. Thornton JG, Hornbuckle J, Vail A, et al. GRIT study group. Infant wellbeing at 2 years of age in the Growth Restriction Intervention Trial (GRIT): multicentred randomised controlled trial. Lancet 2005;364:513-20.

36. Universities Australia, National Health and Medical Research Council, Australian Research Council. Australian Code for the
Responsible Conduct of Research: Australian Government, 2007:1-34.

37. Watanabe K, Hamada F, Wakatsuki A, et al. Prophylactic administration of melatonin to the mother throughout pregnancy can protect against oxidative cerebral damage in neonatal rats. J Matern Fetal Neonatal Med 2012;25:1254-9. 